\title{
Morfometría geométrica en puntas de proyectil lanceoladas de las Sierras Centrales, Argentina
}

\author{
Geometric morphometrics in lanceolate projectile \\ points from Central Hills, Argentina \\ Diego Rivero* y Guillermo Heider**
}

VI Jornadas

Arqueológicas Cuyanas

*CONICET. Centro de Estudios Históricos "Prof. Carlos S. A. Segreti". E-mail: ayampitin1@yahoo.com.ar

${ }^{* *}$ CONICET. Departamento de Geología; Facultad de Cs. Físico, Matemáticas y Naturales; Universidad Nacional de San Luis E-mail: guillermoheider@hotmail.com.

\begin{abstract}
Resumen
Los resultados que se exponen y discuten en este trabajo constituyen un acercamiento inicial a la utilización de la morfometría geométrica como metodología aplicada al estudio de puntas de proyectil lanceoladas de las Sierras Centrales. Este tipo de instrumentos líticos pertenecientes a los sistemas de armas utilizados por los cazadores recolectores, comúnmente denominadas puntas "ayampitín", constituyen un elemento diagnóstico de la tecnología existente en el área durante el Holoceno Medio. El análisis de variables morfométricas y su relación con la materia prima empleada permitió abordar la historia de vida de los artefactos y las variables de diseño existentes, reflejando una amplia variabilidad. El conjunto de cuarenta piezas es el primero al que se aplica esta metodología, por cuanto los resultados obtenidos son preliminares.
\end{abstract}

Palabras clave: Sierras Centrales; Morfometría Geométrica; Puntas de Proyectil Lanceoladas; Holoceno Tempranomedio; Variabilidad.

\begin{abstract}
First results of geometric morphometric techniques applied to a sample of lanceolate projectile points are presented in this paper. These lanceolate projectile points, also known as "ayampitin", are common in the Central Mountains of Argentina and they are usually associated with hunter-gatherer occupations dated to the Middle Holocene. The analyses of morphometric variables and its relation with the selected lithic raw material allowed the analyses of the life history of the artifacts and the existent design variables, reflecting a large variability. This assemblage of forty pieces is the first to be analyzed with these techniques and, therefore, results are preliminary.
\end{abstract}

\section{Keywords}

Central Hills; Geometric Morphometric; Lanceolate Projectiles Points; Early-middle Holocene; Variability.

Las ocupaciones humanas iniciales en Sierras Centrales fueron ubicadas entre ca. 11000 y 9300 AP por diversos investigadores. Las evidencias sobre este período son aún escasas en sitios estratificados. En este sentido, solo los fechados obtenidos en el componente inferior de El Alto 3 (11010 \pm 80 AP LP-1506, $9790 \pm 60$ AP LP-1420, ambos sobre madera carbonizada) y la gruta de Candonga (10450 \pm 50 AP SRLA-1062 sobre hueso humano) pueden ser postulados como propios del tardiglaciar (Rivero 2009; Cornero et al. 2014). A estas evidencias se suman cinco fragmentos de puntas de proyectil "Cola de Pescado" recuperadas en superficie, consideradas como elemento tecnológico diagnóstico para este rango temporal (Schobinger 1974; Laguens et al. 2007; Rivero et al. 2015).
La señal arqueológica regional presenta un considerable aumento a partir del 8.000 AP, periodo a partir del cual se propuso una nueva colonización y posterior ocupación efectiva de los diferentes sectores serranos (Rivero 2012). Existe relativo consenso acerca de considerar las puntas "Ayampitín" como elemento tecnológico diagnóstico para este momento cronológico. Estas fueron halladas en sectores andinos y extra-andinos del centro de Argentina y Chile con una ubicación temporal en un período de 3.000 años, entre 8000 AP y 5000 años AP (v.g. Berberián y Calandra 1984; Gambier 1974). En las Sierras Centrales, su presencia fue identificada y acotada temporalmente por González (1960) al crear la primera secuencia cronológico-cultural de la región. Desde entonces, fueron 


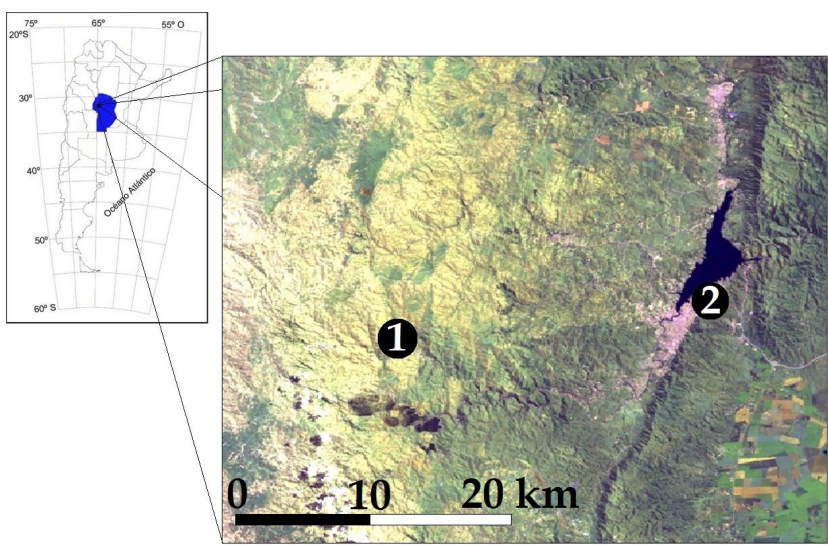

Figura 1: Área de estudio

Figure 1: Study area

utilizadas como artefactos diagnósticos para discutir temporalidad de presencia humana, contextualizando cronológicamente conjuntos arqueológicos no datados (v.g. Sario 2008; Rivero 2009; Pautassi 2011).

En este trabajo se aborda el estudio de las puntas lanceoladas desde una perspectiva diferente a las aplicadas hasta el momento, realizándose un primer análisis desde la morfometría geométrica a un conjunto recuperado en el Valle de Punilla y Pampa de Achala, Córdoba (v.g. Bookstein 1982; Mitteroecker y Gunz 2009; Torcida y Perez 2012). Estudios similares fueron aplicados a puntas de proyectil en distintos contextos arqueológicos de Argentina y Sudamérica (Cardillo 2006, 2009, 2010; Franco et. al. 2009; Cardillo y Charlín 2010; Castiñeira et al. 2011; Charlín y Gónzalez-José 2012; Okumura y Araujo 2014; entre otros).

El objetivo principal de esta publicación es dar a conocer una primera experiencia de trabajo con esta metodología novedosa para el centro del país. El foco está puesto, en este caso, en el diseño y la historia de vida de las puntas ayampitín. Los resultados obtenidos constituyen un paso inicial, cuyo objetivo ulterior será aportar nuevas evidencias para la discusión de las problemáticas de mayor vigencia en el área (poblamiento, fuentes y circulación de materias prima, tecnología lítica, entre otras).

\section{Materiales y métodos}

\section{Muestras}

El conjunto de puntas de proyectil lanceoladas utilizada en este estudio está compuesto por 40 especímenes enteros de diferente origen, 9 de los cuales fueron recuperados en posición estratigráfica en el sitio El Alto 3 y asociados a una datación de $7108 \pm 74$ AP (AA68145 sobre madera carbonizada; Rivero 2009). Las piezas restantes $(n=31)$ provienen de la colección arqueológica del museo Numba-Charaba del Municipio de Villa Carlos

\begin{tabular}{cccc}
\hline Tipo de puntas & Lago San Roque & El Alto 3 & Total \\
\hline Pedunculadas & 6 & 5 & 11 \\
Apedunculadas & 25 & 4 & 29 \\
\hline
\end{tabular}

Tabla 1: Tipos de puntas de proyectil y áreas de procedencia.

Table 1: Types of projectile points and origin areas.

Paz, recolectada en las costas del Lago San Roque (Figura 1 y Tabla 1).

De manera inicial se realizó un estudio tecno-tipológico (Aschero 1975-83) el cual incluyó, además, la identificación de las materias primas sobre las que fueron elaboradas (realizada por el Dr. Edgardo Baldo): el 72,5 \% $(n=29)$ son de cuarzo; el resto se dividen en sílice microcristalino $12,5 \%(n=5)$; calcedonia 2 ( $n=5 \%)$; ópalo, pórfido, basalto $(2,5 \%, n=1)$ e indeterminada $2,5 \%$ en cada caso $(n=1$ $\mathrm{c} / \mathrm{u}$ ). Las rocas seleccionadas como soporte son de buena calidad para la talla, sin embargo tienen diferencias en sus aptitudes respecto a la predictibilidad de su fractura. Por otra parte, poseen disponibilidad diferencial en el paisaje, siendo el cuarzo una roca presente de forma ubicua en el área mientras que las restantes materias primas están a distancias superiores a los $50 \mathrm{~km}$ de los sitios considerados (Heider et al. 2015).

\section{Técnicas morfométricas en puntas de proyectil}

La descripción de la variación de la forma entre las puntas estudiadas fue realizada mediante el empleo de técnicas de la morfometría geométrica y métodos estadísticos multivariados. Para la obtención de los datos morfométricos, se tomaron fotos digitales de todos los ejemplares a una distancia de $30 \mathrm{~cm}$ con una escala localizada en el costado izquierdo de los mismos. Las fotografías se compilaron en el programa TpsUtil (ver.1.26 Rohlf 2004a), luego se digitalizaron los puntos morfométricos en el TpsDig (ver.1.4 Rohlf 2004b) ubicando dos landmarks (en el ápice y el punto de la base más alejado de éste) y 10 semilandmarks por cada borde (20 en total).

La morfometría geométrica es una técnica (común en estudios biológicos) cuyo uso se ha incrementado de manera reciente en arqueología. La misma permite obtener una descripción cuantitativa de la forma en espacios bidimensionales o tridimensionales, a partir del estudio de las relaciones espaciales entre puntos morfométricos homólogos (v.g. Bookstein 1982; Mitteroecker y Gunz 2009; Torcida y Pérez 2012; Gunz y Mitteroecker 2013). Los puntos de referencia o "landmarks" son geométricamente homólogos entre estructuras. Existen tres tipos de landmarks (Bookstein 1991), el Tipo I es el de mayor evidencia de homología, el Tipo II se corresponde con puntos cuya homología sólo se sostiene con evidencia geométrica (v.g. puntos 
de máxima curvatura, el ápice de las puntas de proyectil) y, finalmente, el Tipo III son puntos con al menos una coordenada ambigua que no son considerados homólogos y caracterizan más de una región de la estructura (v.g. puntos arbitrarios posicionados sobre el contorno de la forma). Debido a la naturaleza ambigua de los landmarks Tipo III, fue revisada su clasificación y se los denominó "semilandmarks" (Bookstein 1997).

El empleo de semilandmarks es adecuado cuando las estructuras en estudio poseen pocos puntos discretos o éstos están concentrados en una región de la estructura. Este es el caso del análisis de las puntas de proyectil, que en nuestro caso sólo poseen dos puntos homólogos (el ápice y el punto más alejado de la base orientados según el eje morfológico de la pieza) por lo que es necesario el uso de semilandmarks para describir adecuadamente la forma de estos artefactos. Esta descripción es, por otra parte, independiente del volumen efectivo del objeto por cuanto tamaño y forma pueden ser analizadas de manera independiente, eliminando el efecto del tamaño, traslación y rotación para lograr una minimización de las diferencias en las configuraciones de landmarks (Rohlf y Slice 1990; Zelditch et al. 2004). Un procedimiento eficiente para minimizar las diferencias entre especímenes y la configuración de consenso (que constituye la forma con respecto a la cual las distintas configuraciones de forma se encuentran lo más cerca posible) corresponde al método conocido como Análisis de Procrustes Generalizado (APG). El método para eliminar las diferencias de tamaño en este trabajo es de Procrustes, que minimiza las diferencias entre las formas originales y una morfología media o forma de consenso, que se utiliza para la superposición (Rohlf 1990; Torcida y Pérez 2012). El análisis de Procrustes permite comparar configuraciones de puntos homólogos provenientes de variantes de la misma entidad, es decir individuos de la misma población. Este método es apropiado para estudiar diferencias de forma entre estructuras representadas por sus configuraciones de landmarks (Torcida y Pérez 2012). Debido a que los semilandmarks no son puntos homólogos, es necesario deslizarlos (sliding) para poder darles un espaciamiento regular previo a la superposición Procrustes. Para ello se siguió el criterio propuesto por Perez et al. (2006), mediante la distancia Procrustes con respecto a la forma consenso. Para la realización del análisis Procrustes generalizado y el deslizamiento de los semilandmarks, se utilizó el TpsRelw (ver.1.54 Rohlf 2014).

\section{Análisis multivariados}

La variación en forma entre las puntas de proyectil fue explorada, en primer lugar, empleando la técnica de componentes principales. Este es un método para reducir un gran conjunto de variables a unas pocas dimensiones que representan la mayor parte de la variación en los datos, perdiendo la menor cantidad de información posible. Para reducir la dimensión de los datos (en este caso coordenadas Procrustes) se realiza la rotación de los ejes para obtener nuevos ejes ortogonales y no correlacionados entre sí (Componentes Principales), de manera que el primero de ellos contenga la mayor variación de la muestra, seguido por otro eje que contenga la segunda cantidad de variación de la muestra y así sucesivamente (Mitteroecker y Gunz 2009). Los componentes principales corresponden a los autovalores de la matriz de covarianza de las coordenadas Procrustes, y aquellos con valores más altos explican la mayor parte de la variación observada y pueden proyectarse en un gráfico de dos dimensiones, donde el eje principal corresponde al CP1 y el restante al CP2, y permite visualizar diferencias, tendencias y agrupamientos de los especímenes analizados (Mitteroecker y Gunz 2009). Finalmente, se exploró la covariación entre la forma y las otras variables de interés. Para esto se empleó la técnica de Partial Least Squares (PLS) y análisis discriminante o de variable canónica (CVA). PLS es un método estadístico que permite explorar patrones de covariación entre dos conjuntos de variables continuas, difiriendo de otros análisis de regresión en que las variables son tratadas simétricamente para hallar relaciones entre ellas sin asumir que una es la causa de variación de la otra (Rohlf y Corti 2000).

Tal como lo mencionamos, la asociación entre la forma de las puntas de proyectil y la materia prima utilizada en su manufactura fue explorada empleando un análisis de variables canónicas (CVA). La misma es una técnica de clasificación multivariada en la cual se realiza una extensión del análisis discriminante a múltiples grupos optimizando un conjunto de relaciones análogo (Klingenberg y Monteiro 2005; Mitteroecker y Bookstein 2011). En este caso, las variables clasificatorias son la materia prima y la presencia de pedúnculo, siendo la primera seleccionada con el fin de determinar si es capaz de explica una parte significativa de la varianza morfológica. Para los análisis de estadística multivariada de componentes principales Partial Least Squares y de variables canónicas, se utilizó MorphoJ (Klingenberg 2009). Se importaron las coordenadas Procrustes y el tamaño centroide obtenidos en el TpsRelw agregándose variables no geométricas (v.g. espesor, presencia/ausencia de pedúnculo y tipo de materia prima), para los análisis multivariados mencionados.

\section{Resultados}

El análisis indica que los dos principales componentes explican el $65 \%$ de la variación morfológica, correspondiendo al primer componente el $47 \%$ donde se observan cambios en el alargamiento y ancho de las piezas. En tanto que el segundo componente explica el $18 \%$ de la variación, indicando cambios relacionados con la presencia de pedúnculo (Figura 2).

En la amplia variabilidad observada a ojo desnudo se 

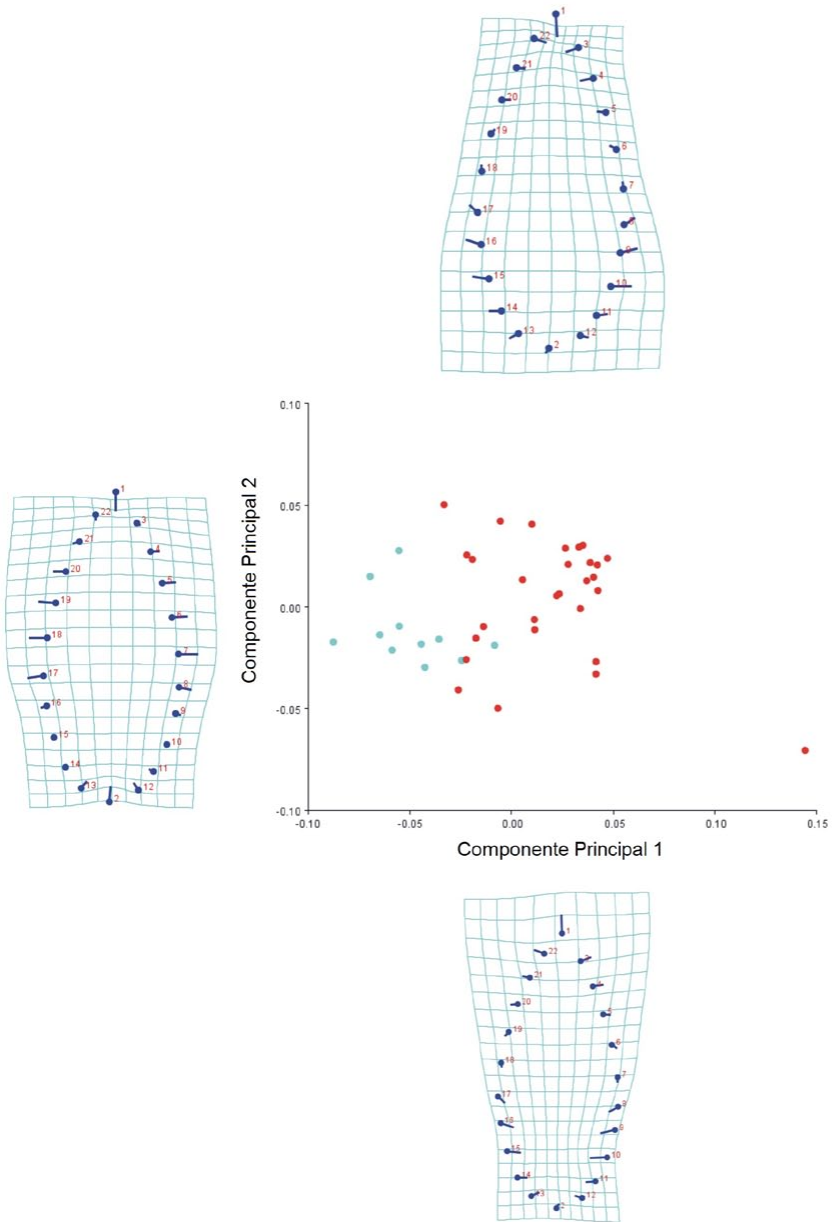

Figura 2: Análisis de Componentes Principales y plantillas de deformación. En celeste los ejemplares pedunculados y en rojo los apedunculados. El componente uno explica el $47 \%$ del conjunto total; el componente dos explica el $18 \%$ del conjunto total.

Figure 2: Principal Component Analysis and deformation templates. Light blue: Pedunculated projectile points; red: Non-pedunculated projectile points. Component one explains $47 \%$ of the total sample; component two explains $18 \%$ of the total sample.

destacan, al menos, dos tipos morfológicos básicos (TMB sensu Martínez 2003), uno lanceolado apedunculado y otro lanceolado pedunculado. Dentro de cada uno de estos TMB existe una cierta variabilidad en tamaño (alargados/ angostos vs. cortos/anchos), a la que se suman algunos ejemplares que presentan un denticulado en el limbo. Por su parte, el análisis Partial Least Squares (Rohlf 1990) indica una moderada, aunque significativa, correlación $(r=0,50447 ; p=0,0279)$ entre el logaritmo del Tamaño Centroide (definido como la raíz cuadrada de la suma de las distancias cuadradas de todos los puntos desde su centro de gravedad, Mitteroecker y Gunz 2009) y las coordenadas Procrustes. Se manifiesta principalmente en el sector mesial y apical de los ejemplares, percibiéndose un cambio entre formas más alargadas/angostas para los tamaños más grandes y formas más cortas/anchas para los tamaños menores que incluyen los ejemplares con pedúnculo esbozado. Esto indicaría que las formas más pequeñas no son resultado de la reactivación de un diseño estandarizado representado por las formas más grandes (Figura 3).

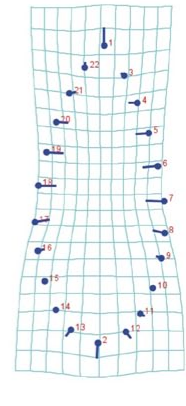

En cuanto a la influencia de la materia prima sobre la variación morfológica observada, la misma se evaluó mediante un análisis de variable canónica. El mismo indicó que las dos primeras variables canónicas (VC) explican el $70 ., 47 \%$ de la variación observada, correspondiendo el 40,62 \% a la primera variable (VC1) y el $29,85 \%$ restante a la segunda variable (VC2) (Figura 4). Se observan diferencias leves, aunque significativas $(p<0,05)$, entre algunas materias primas empleadas (v.g. cuarzo, sílice microcristalino, calcedonia y ópalo) y las variables de forma (Tabla 2). Las principales diferencias se observan en los valores positivos de la VC2, vinculados con la presencia de pedúnculo esbozado en los ejemplares de sílice microcristalino y calcedonia, como puede observarse en las plantillas de deformación (Figura 4).

\section{Discusión y perspectivas futuras}

Las puntas de proyectil lanceoladas constituyen artefactos con características técnicas reconocibles y relativamente acotadas temporalmente en la arqueología del Centro-oeste del país en general y de las Sierras Centrales en particular. Los abordajes utilizados para su estudio estuvieron ligados, hasta el momento, a su ubicación temporal en contextos arqueológicos y cuestiones tecnológicas (v.g. Rivero 2009; Pautassi 2011). En este trabajo se utilizó una nueva metodología para caracterizar a las ayampitín y entender la variabilidad morfológica dentro de este "tipo" de punta de proyectil. Los resultados representan un punto de partida, tanto para la realización de abordajes de mayor complejidad en el área como así también para comparación futura con problemáticas relacionadas en otros sectores del país donde la metodología lleva ya más de una década de aplicación y discusión (Cardillo 2006, 2010, Ratto 2012, entre otros).

De manera general, puede mencionarse que las puntas lanceoladas se caracterizan por su variabilidad, al menos en el sector de las Sierras Centrales estudiado. Al respecto, Pautassi (2011) desarrolla un detallado análisis en el cual identifica tres diseños particulares y diferenciables entre sí (Clases A, B y C) a los cuales les atribuye diferencias surgidas de su manufactura para formar parte de sistemas de armas distintos (v.g. lanza, propulsor) especialmente la clase $C$ y determina que "...la variabilidad no obedece a distribuciones espaciales particulares producidas a escala regional" (Pautassi 2011: 129). Con este panorama 

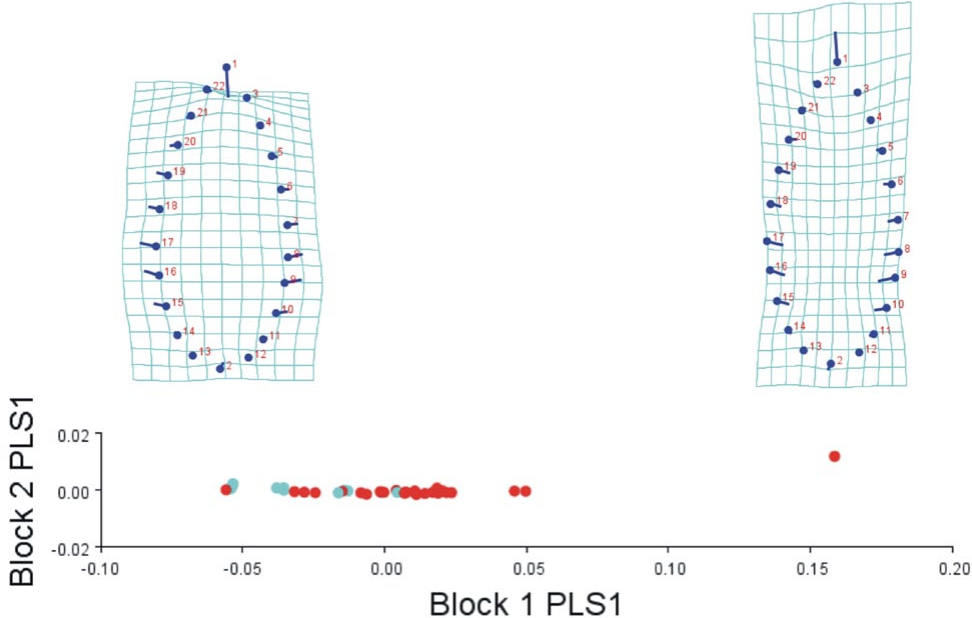

Figura 3: Resultado del PLS y grillas de deformación. En celeste los ejemplares pedunculados $(27,5 \%)$ y en rojo los apedunculados (72,5\%).

Figure 3: Result of PLS and deformation grids. Light blue: Pedunculated projectile points $(27,5 \%)$; red: Non-pedunculated projectile points $(72,5 \%)$.

Tabla 2: Valor $\mathrm{P}$ del test de permutación para las distancias Mahalanobis entre pares de materia prima.

Table 2: P-values of the permutation test for the Mahalanobis distances between pairs of lithic raw material.

\begin{tabular}{lcccccc}
\hline & Basalto & Calcedonia & Cuarzo & Indeterm & Pórfiro & Sílice Mic. \\
\hline 2. Calcedonia & 0.3336 & - & - & - & - & - \\
3. Cuarzo & $\mathbf{0 . 0 2 7 7}$ & $\mathbf{0 . 0 0 1 9}$ & - & - & - & - \\
4. Indeterm. & 1.0000 & 0.3355 & $\mathbf{0 . 0 0 2 8}$ & - & - & - \\
5. Pórfiro & $<.0001$ & 0.3272 & $\mathbf{0 . 0 0 8 0}$ & 1.0000 & - & - \\
6. Sílice Mic. & 0.1024 & $\mathbf{0 . 0 3 2 4}$ & $<.0001$ & $\mathbf{0 . 0 4 3 9}$ & 0.1557 & - \\
Ópalo & $<.0001$ & $<.0001$ & 0.0024 & 1.0000 & 1.0000 & 0.0931 \\
\hline
\end{tabular}

Figura 4: Variables canónicas y plantillas de deformación según la materia prima. La VC1 explica el 40,62\% del total, la CV2 explica el $29,85 \%$ del total.

Figure 4: Canonical variables and deformation templates according to the lithic raw material. The VC1 explains $40,62 \%$ of the total sample; CV2 explains $29,85 \%$ of the total sample.
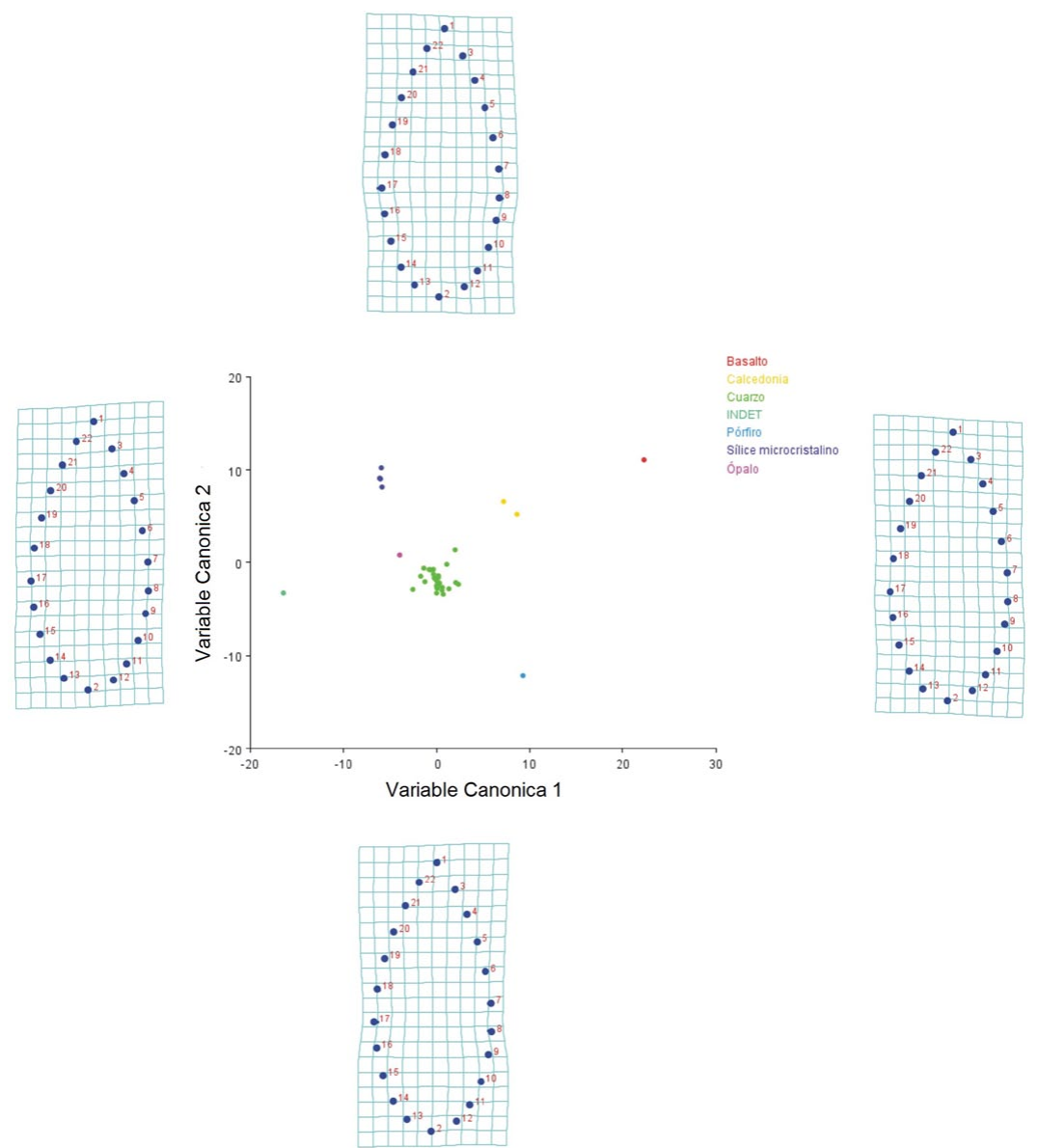
de variabilidad, propuesto por Pautassi y corroborado nuevamente en este trabajo (v.g. análisis de PLS), pueden trazase algunas líneas a explorar.

Se sostiene, que la elección de la materia prima está vinculada a la disponibilidad local y/o la accesibilidad a fuentes lejanas (Heider et al. 2015). A partir del estudio morfométrico se pudo observar la existencia de una influencia (baja pero significativa) de las materias primas respecto de las formas. Esto se evidenció en algunos ejemplares de sílice/calcedonia que poseen pedúnculo esbozado y podría estar relacionado con una fractura más predecible para la talla en este tipo de rocas. El resultado observado no invalida las posturas previamente mencionadas, por el contrario resulta un aporte novedoso que incrementa el espectro de información para evaluar los postulados vigentes.

Otro aspecto donde la morfometría podría aportar una nueva línea de evidencia es al modelo de poblamiento de la región. Para comprender la dinámica en los modos de vida de los cazadores-recolectores se debe profundizar en aspectos biogeográficos, paleoambientales, ideológicos y económicos, las cuales sin duda influyeron en la toma de decisiones en los momentos de exploración, colonización y ocupación efectiva de las Sierras Centrales. Por otra parte, surgen nuevas líneas a desarrollar, en donde la morfometría será de gran utilidad, como la manufactura y mantenimiento de sistemas de armas o predictibilidad de fractura. En este marco, el primer avance a partir de la morfometría geométrica y el análisis multivariado se ha revelado como una técnica de análisis valiosa sobre la cultura material de los pueblos originarios cuyo aporte será cada vez de mayor relevancia para discutir diferentes líneas de trabajo en el centro del país.

Córdoba, 18 de noviembre de 2015

\section{Agradecimientos}

Queremos agradecer a Iván Pérez por su guía para la aplicación de las técnicas de la morfometría geométrica y estadística multivariada. A Silvia Ledda por permitirnos analizar las piezas de museo incluidas en este análisis y Sebastián Pastor por la gestión realizada con tal fin. Al Dr. Eduardo Berberián y a nuestros compañeros del Área de Arqueología del C.E.H. por el apoyo constante. A los evaluadores por sus correcciones que mejoraron notoriamente este primer acercamiento a la temática. Esta investigación fue financiada por CONICET (PIP 112200801-02678).

\section{Bibliografía}

Aschero, C. 1975-1983. Ensayo para una clasificación morfológica de artefactos líticos. Informe a CONICET. Ms.

Berberián, E. \& H. Calandra 1984. Investigaciones arqueológicas en El Peñoncito, San Juan, República Argentina. Revista del Museo de La Plata VIII (56):139169.

Bookstein, F. 1982. Foundation of Morphometrics. Annual Review of Ecology and Systematics 12: 451-470.

Bookstein, F. 1991. Morphometric Tools for Landmark Data: Geometry and Biology. Cambridge University Press. Cambridge.

Bookstein, F. 1997. Landmark methods for forms without landmarks: Localizing group differences in outline shape. Medical Image Analysis. 1 pp. 225-243

Cardillo, M. 2006. Explorando la variación en las morfologías líticas a partir de las técnicas de análisis de contornos. El caso de puntas de proyectil del Holoceno Medio-Tardío de la Puna de Salta (San Antonio de los Cobres, Argentina).Werken Vol. 7.

Cardillo, M. 2009. Temporal trends in the morphometric variation of the lithic projectile points during the Middle Holocene of southern Andes (Puna region). A Coevolutionary approach. Theoretical and methodological issues in evolutionary archaeology: toward an unified darwinian paradigm. SAMAR series. Vol. 20, pp 13-20.

Cardillo, M. 2010. Some Applications of Geometric Morphometrics to Archaeology. Ashraf M. y T. Elewa (eds.) Morphometrics to Nonmorphometricians, 235241, Springer-Verlag. Berlín.

Cardillo, M. \& J. Charlin, 2010. Tendencias observadas en la variabilidad de los raspadores de norte y sur de Patagonia. Explorando las interrelaciones entre forma, tamaño e historia de vida. 2do Congreso Argentino y 1 er Congreso Latinoamericano de Arqueometría Vol.2: 351-359. Buenos Aires

Castiñeira, C., M. Cardillo, J. Charlin \& J. Baeza. 2011. Análisis de morfometría geométrica en cola de pescado del Uruguay. Latin American Antiquity 22 (3): 335-358.

Charlin, J \& R. González-José, 2012. Size and shape variation in Late Holocene projectile points of Southern Patagonia: a geometric morphometric study. American Antiquity 77 (2), 221-242.

Cornero, S., W. Neves \& R. Rivero. 2014. Nuevos aportes a la cronología de las ocupaciones tempranas en las Sierras de Córdoba. La gruta de Candonga (Córdoba, Argentina). Relaciones de la Sociedad Argentina de Antropología XXXIX (1): 285-292.

Franco, N., A. Castro, M. Cardillo \& J. Charlin. 2009. La importancia de las variables morfológicas, métricas y de 
microdesgaste para evaluar las diferencias en diseños de puntas de proyectil bifaciales pedunculadas: un ejemplo del sur de Patagonia continental. Magallania 37(1): 99-112.

Gambier, M. 1974. Horizonte de Cazadores Tempranos en los Andes Centrales argentino-chilenos. Hunuc-huar 2:43-103.

González, A. 1960. La estratigrafía de la gruta de Intihuasi (Prov. de San Luis, R.A.) y sus relaciones con otros sitios precerámicos de Sudamérica. Revista del Instituto de Antropología 1: 5-296.

Gunz, P. \& P., Mitteroecker. 2013. Semilandmarks: a method for quantifying curves and surfaces. Hystrix 24, 103-109.

Heider, G., Rivero, D. \& E. Baldo. 2015. Rocas de uso arqueológico en Sierras Centrales. Fuentes de recursos líticos identificadas y potenciales en las provincias de Córdoba y San Luis, Argentina. Revista de Antropología del Museo de Entre Ríos 1 (2): 55-72.

Klingenberg, C. 2009. MorphoJ: an integrated software package for geometric morphometrics. Mol. Ecol. Resour. 11:353-357.

Klingenberg, C. \& Monteiro, L.R., 2005. Distances and directions in multidimensional shape spaces: implications for morphometric applications. Syst. Biol. 54:678-688.

Laguens, A., E. Pautassi, G. Sario y R. Cattáneo. 2007. ELS1, a Fishtail Projectile Point Site from Central Argentina. Current Research in the Pleistocene 24:55-57.

Martínez, J. 2003. Ocupaciones Humanas Tempranas y Tecnología de Caza en la Microrregión de Antofagasta de la Sierra (10000-7000 AP). Tesis de Doctorado. Facultad de Ciencias Naturales e Instituto Miguel Lillo. U. N. T. ms.

Mitteroecker P. \&F. Bookstein F, 2011. Linear Discrimination, Ordination, and the Visualiza-tion of Selection Gradients in Modern Morphometrics. Evolutionary Biology 39: 100-114.

Mitteroecker P. \& P. Gunz. 2009. Advances in Geometric Morphometrics. Evol Biol 36:235-247.

Okumura, M. \& A. Araujo 2014. Long-term cultural stability in hunter-gatherers: a case study using traditional and geometric morphometric analysis of lithic stemmed bifacial points from Southern Brazil. Journal of Archaelogical Science 45: 59-71

Pautassi, E. 2011.Tecnología de proyectiles, durante el Holoceno temprano, en la porción Austral de las sierras
Pampeanas. Martínez J. y L. Bozzuto (eds.).Armas prehispánicas: múltiples enfoques para su estudio en Sudamérica.115-134. Fundación de Historia Natural Félix de Azara. Buenos Aires.

Pérez, I., Bernal V. \& P. González, 2006. Differences between sliding semilandmarks methods: implications for shape analyses of human populations. Journal of Anatomy 208:769-784.

Ratto, N. 2012. Diversidad de tecnología de caza en la Puna transicional de Chaschuil (Dpto. Tinogasta, Catamarca). Comechingonia 17 (1): 83-101.

Rivero, R. 2012. La ocupación humana durante la transición Pleistoceno-holoceno (11,000 - 9,000 a.P) en las Sierras Centrales de Argentina. Latin American Antiquity 23 (4): 551-564

Rivero, D. 2009. Ecología de cazadores-recolectores del sector central de las Sierras de Córdoba (Rep. Argentina). BAR International Series 2007, Oxford.

Rivero, D. 2012. La ocupación humana durante la transición Pleistoceno-Holoceno (11,000 - 9,000 a.P.) en las Sierras Centrales de Argentina. Latin American Antiquity 23(4):551-564.

Rivero, D., G. Heider \& S. Pastor. 2015. Identificación de una punta cola de pescado en las Sierras de Córdoba. Implicancias para el poblamiento del Centro de Argentina. Cuadernos del Instituto de Antropología y Pensamiento Latinoamericano. En prensa.

Rohlf, F. 1990. Rotational fit (Procrustes) methods. En Proceedings of the Michigan Morphometrics Workshop (F. J. Rohlf y F. L. Bookstein, eds.) Special Publ. No. 2. The University of Michigan Museum of Zoology. Pp. 227-236.

Rohlf, F. 2004a. TpsUtil, file utility program. Version 1.26. Department of Ecology and Evolution, State University. Stony Brook, New York.

Rohlf, F. 2004b. TpsDig, version 1.4. Department of Ecology and Evolution, State University. Stony Brook, New York.

Rohlf, F. 2014. TpsRelw, version 1.54. Department of Ecology and Evolution, State University. Stony Brook, New York.

Rohlf, F. \& D. Slice, 1990. Extensions of the Procrustes method for the optimal superimposition of landmarks. Systematic Zoology 39 (1):40-59.

Rohlf, F. \& M. Corti, 2000. Use of two-block partial least-squares to study covariation in shape. Syst. Biol., 
49(4):740-53.

Sario, G. 2008. Tecnología Bifacial en las Sierras de San Luis y depresión del Conlara (Provincia de San Luis, República Argentina) en el Holoceno Temprano. Arqueoweb Revista sobre Arqueológia en Internet 10:1-18

Schobinger, J. 1974. Nuevos hallazgos de "Cola de Pescado" y consideraciones en torno al origen y dispersión de la Cultura de Cazadores Superiores Toldense en
Sudamérica. Atti del XL Congreso Internazionale degle Americanisti 1:33-50, Roma-Génova.

Torcida, S. \& I. Pérez, 2012. Análisis de Procrustes y el estudio de la variación morfológica. Revista Argentina de Antropología Biológica 14: 131-141.

Zelditch, M., D. Swiderski, H. Sheets \& W. Fink. 2004. Geometric morphometric for biologists: a Primer. Academic Press. Londres 